\title{
$Q$ fever endocarditis: A case report and review of the literature
}

\author{
Marc W Deyell MD ${ }^{1}$, Brian Chiu MD², David B Ross MD³ ${ }^{3}$ Nanette Alvarez MD
}

MW Deyell, B Chiu, DB Ross, N Alvarez. Q fever endocarditis: A case report and review of the literature. Can J Cardiol 2006;22(9):781-785.

The case of a 31-year-old man from Alberta diagnosed with $Q$ fever endocarditis is presented. To the authors' knowledge, this is the first case of $Q$ fever endocarditis diagnosed in the province of Alberta. The patient had undergone open valvulotomy for congenital aortic stenosis as an infant. He presented with congestive heart failure secondary to severe aortic regurgitation and underwent mechanical aortic valve replacement. Early failure of the mechanical prosthesis and numerous laboratory abnormalities prompted an investigation for endocarditis, which was initially negative. Markedly positive serology eventually established the diagnosis of chronic $Q$ fever. The patient subsequently underwent a second aortic valve replacement following initiation of appropriate antimicrobials directed against Coxiella burnetii. The present report reviews the clinical presentation and diagnosis of $Q$ fever endocarditis. It highlights the insidious and nonspecific nature of the presenting symptoms, and emphasizes the use of serology for diagnosis. Increased awareness and earlier diagnosis can significantly decrease the morbidity and mortality associated with this disease.

\section{L'endocardite de la fièvre $Q$ : Rapport de cas et analyse bibliographique}

Le cas d'un Albertain de 31 ans obtenant un diagnostic d'endocardite de la fièvre $Q$ est exposé. En autant que le sache l'auteur, c'est le premier cas d'endocardite de la fièvre $Q$ à être diagnostiqué dans la province de l'Alberta. Lorsqu'il était nourrisson, le patient a subi une valvulotomie ouverte en raison d'une sténose aortique congénitale. Il a consulté parce qu'il souffrait d'insuffisance cardiaque congestive secondaire à une régurgitation aortique marquée. Il a subi un remplacement valvulaire mécanique de l'aorte. Un échec rapide de la prothèse mécanique et de nombreuses anomalies de laboratoire ont suscité une exploration d'endocardite, qui s'est d'abord révélée négative. Une sérologie manifestement positive a fini par permettre d'établir le diagnostic de fièvre $\mathrm{Q}$ chronique. Le patient a subi un deuxième remplacement valvulaire aortique après l'application d'un traitement antimicrobien orienté contre le Coxiella burnetii. Le présent rapport analyse la présentation clinique et le diagnostic d'endocardite de la fièvre. Il souligne la nature insidieuse et non spécifique des manifestations initiales ainsi que l'utilisation de la sérologie pour poser le diagnostic. Une meilleure sensibilisation et un diagnostic plus précoce peuvent réduire considérablement la morbidité et la mortalité associées à cette maladie.

Key Words: Coxiella burnetii; Endocarditis; Q Fever

Cndocarditis is the most common presentation of chronic EQ fever, a zoonosis caused by the obligate intracellular bacteria Coxiella burnetii. Commonly believed to be a rare disorder, it has been estimated to account for up to $5 \%$ of all endocarditis cases worldwide (1-3). It occurs almost exclusively in patients who have pre-existing valvular disease or who are immunocompromised (4). Unlike typical cases of endocarditis, the clinical presentation of chronic $Q$ fever is often nonspecific. Furthermore, specialized techniques are required for identification of the organism, and echocardiographic evidence of infection is frequently absent (5). Without prompt recognition and appropriate antimicrobial therapy, the course of $Q$ fever endocarditis is severe and potentially fatal. The following case demonstrates many of the clinical features of chronic $Q$ fever and highlights the difficulty of obtaining the diagnosis.

\section{CASE PRESENTATION}

A 31-year-old Caucasian man presented to a walk-in clinic complaining of progressive, exertional shortness of breath. He had moved to Calgary, Alberta, from Nova Scotia, where he had worked on a dairy farm, two years prior. Due to pre-existing congenital valvular heart disease, he was referred to the Adult Congenital Cardiology Clinic at the University of Calgary (Calgary, Alberta). The patient could barely climb one flight of stairs without significant shortness of breath, and had developed orthopnea and paroxysmal nocturnal dyspnea. He described occasional palpitations, but denied any syncopal episodes, chest discomfort or peripheral edema. There were no symptoms suggestive of systemic infection.

Of note, the patient had been diagnosed with congenital aortic stenosis at the age of eight months and underwent open aortic valvulotomy. The patient was found to have a trileaflet valve with significant fusion of the leaflets. Catheterization at the age of 10 years revealed a peak residual gradient of $25 \mathrm{mmHg}$ across the aortic valve with mild aortic insufficiency. Echocardiograms at the ages of 18 and 22 years demonstrated peak gradients of $37 \mathrm{mmHg}$ and $56 \mathrm{mmHg}$, respectively, moderate aortic insufficiency and preserved left ventricular function. The patient had no cardiology follow-up after the age of 22 years.

Physical examination revealed a blood pressure of $110 / 50 \mathrm{mmHg}$, a heart rate of 82 beats/min and a normal

${ }^{1}$ Division of General Internal Medicine, Department of Internal Medicine, University of Calgary, Calgary; ${ }^{2}$ Division of Anatomical Pathology,

Department of Laboratory Medicine and Pathology; ${ }^{3}$ Division of Cardiac Surgery, Department of Surgery, University of Alberta, Edmonton;

${ }^{4}$ Division of Cardiology, Department of Cardiac Sciences, University of Calgary, Calgary, Alberta

Correspondence: Dr Nanette Alvarez, Department of Cardiovascular Services, Peter Lougheed Centre, 3500 - 26th Avenue Northeast, Calgary,

Alberta T1Y 6J4. Telephone 403-943-4920, fax 403-250-9539, e-mail diane.thomas@calgaryhealthregion.ca

Received for publication August 5, 2005. Accepted December 22, 2005 
oxygen saturation on room air. He was cachectic and had severe acne. No peripheral stigmata of endocarditis were found. Bounding pulses were evident along with a positive Durozier's sign. The patient's jugular venous pressure was normal. Auscultation revealed a single S2, as well as an S3. He had a grade 4 of 6 systolic ejection murmur at the base, with a long, grade 3 of 6 , high-pitched, diastolic murmur best heard along the left lower sternal border. The patient's lungs were clear on auscultation. No peripheral edema was evident. Mild hepatosplenomegaly was noted.

The electrocardiogram showed an incomplete left bundle branch block and left ventricular hypertrophy with nonspecific repolarization abnormalities. A transthoracic echocardiogram showed a dilated left ventricle (end-systolic and end-diastolic dimensions of $74 \mathrm{~mm}$ and $51 \mathrm{~mm}$, respectively) with normal systolic function. There was mild aortic stenosis with a peak gradient of $48 \mathrm{mmHg}$, but severe aortic regurgitation (grade 4+) and a peak right ventricular systolic pressure of $55 \mathrm{mmHg}$. No vegetations were identified. A magnetic resonance imaging scan revealed normal origins of both coronaries but slight dilation of the left coronary artery. The aortic root was normal. Cardiac catheterization revealed an ejection fraction of $41 \%$, left ventricular end-diastolic pressure of $29 \mathrm{mmHg}$, severe aortic regurgitation, mild aortic stenosis and normal coronary arteries.

The patient was noted to have a number of laboratory abnormalities, including mild normocytic anemia (hemoglobin $110 \mathrm{~g} / \mathrm{L}$; mean corpuscular volume $87 \mathrm{fL}$ ), mild lymphocytosis $\left(3.7 \times 10^{9}\right.$ cells/L) and moderate neutropenia $\left(1.1 \times 10^{9}\right.$ cells $\left./ \mathrm{L}\right)$. Iron studies were consistent with anemia of chronic disease. A peripheral smear revealed atypical lymphocytes, which were subsequently deemed to be reactive on the basis of a normal bone marrow biopsy. Blood cultures were consistently negative, though his erythrocyte sedimentation rate (ESR) was elevated ( $63 \mathrm{~mm}$; normal range $0 \mathrm{~mm}$ to $10 \mathrm{~mm}$ ). Further testing revealed liver enzyme abnormalities, with an alkaline phosphatase concentration of $154 \mathrm{U} / \mathrm{L}$ (normal $30 \mathrm{U} / \mathrm{L}$ to $145 \mathrm{U} / \mathrm{L}$ ) and an alanine aminotransferase concentration of $99 \mathrm{U} / \mathrm{L}$ (normal $1 \mathrm{U} / \mathrm{L}$ to $60 \mathrm{U} / \mathrm{L}$ ). Serum protein electropheresis was notable for increased gamma globulins consisting of several monoclonal bands. An extensive workup, including consultation with the Department of Internal Medicine and Infectious Diseases, revealed no identifiable cause, and $Q$ fever was not suspected at that time. These findings were attributed to severe aortic insufficiency and subsequent 'cardiac cachexia'.

The patient was referred to the Congenital Cardiovascular Surgery service at the University of Alberta (Edmonton, Alberta). At surgery, the ascending aorta was dilated at $57 \mathrm{~mm}$, but the sinotubular junction and aortic sinuses appeared normal. The aortic valve, subaortic area and anterior mitral valve leaflets looked as though they had previously been affected by endocarditis. There was a deep aneurysm of the perimembranous intraventricular septum consistent with a healed abscess cavity. There were no signs of active infection. Microscopy and cultures of operative specimens revealed no microorganisms. Pathological examination of the resected aortic valve specimen reported marked nodular fibrosis and calcification. He underwent aortic valve replacement with a $29 \mathrm{~mm}$ St Jude mechanical aortic prosthesis (St Jude Medical Inc, USA), replacement of the ascending aorta with a $30 \mathrm{~mm}$ Hemashield graft (Boston Scientific, USA), suture repair of an anterior mitral valve leaflet perforation, and repair of the perimembranous aneurysm of the intraventricular septum.
Follow-up at three months after surgery found the patient feeling well, but examination unexpectedly revealed a grade 3 of 6 diastolic murmur. A transthoracic echocardiogram confirmed a moderate to severe paravalvular leak, which, with persistent lymphocytosis, prompted a repeat infectious disease consultation. Eight sets of blood cultures were obtained and incubated for 21 days. All were negative. Additionally, serology for Brucella species and Legionella species was negative. The cardiac surgeon, who had previously practiced in Nova Scotia, became suspicious that this could be a case of $Q$ fever endocarditis and prompted serology for Coxiella species to be sent as well. The patient was treated empirically with ciprofloxacin and rifampin, given his province of origin and the appearance of his valve at the time of his surgery. Serology for $Q$ fever was delayed due to the concurrent severe acute respiratory syndrome epidemic and the subsequent demands on the provincial microbiological laboratory.

Due to the severity of his cardiac symptoms, the patient underwent urgent surgery, including repeat aortic root replacement with a $25 \mathrm{~mm}$ homograft valve, replacement of the ascending aorta with an aortic homograft and repair of the left ventricular outflow tract aneurysm. Intraoperatively, they found the aortic graft to be encased in thick fibrotic tissue. Histological examination of the valve revealed valvulitis but no vegetations. Special histological stains for microorganisms were negative. Repeated electron microscopy studies failed to show any Coxiella species organisms. Shortly after the operation, the patient's Coxiella species serology returned. The phase I antigen had a titre of 1:131,072, and the phase II titre was $1: 65,536$, consistent with a diagnosis of chronic $Q$ fever. The valve tissue was subsequently sent to the research laboratory of Dr D Raoult in Marseilles, France, where C burnetii was isolated from the valve using special culture techniques.

The patient had a complicated postoperative course, including a right lateral thoracotomy for empyema. He was discharged from hospital six weeks after his second aortic valve replacement. Follow-up at one, three, six and 12 months found the patient doing well, with no evidence of systemic infection and a normal-appearing and normal-functioning aortic valve prosthesis.

\section{DISCUSSION}

$Q$ fever is a ubiquitous infection and has been reported worldwide. There are some areas that appear to have a preponderance for the disease, such as the Basque region of Spain and the south of France (4). Domestic ruminants (cattle, goats and sheep) form the major reservoir for C burnetii. However, domestic cats and dogs may also be reservoirs, as highlighted in a number of outbreak investigations $(6,7)$. Humans are infected by the inhalation of aerosolized organisms following exposure to infected fomites or products of conception. The acute form of $Q$ fever typically presents as a nonspecific febrile illness, acute hepatitis or atypical pneumonia; however, as many as $60 \%$ of cases are asymptomatic (4).

Of particular relevance in Canada, Nova Scotia has a particularly high prevalence of $\mathrm{C}$ burnetii infection, which was first revealed when acute $Q$ fever was found to be a common cause of atypical pneumonia in the province (8). Subsequently, 174 cases were reported by Marrie (9) between 1980 and 1987. Furthermore, 11 cases of $Q$ fever endocarditis were reported in the province from 1979 to 2003 (10). It has been estimated that $\mathrm{C}$ burnetii is the etiological agent in approximately $3 \%$ of 
TABLE 1

Clinical and laboratory manifestations of chronic $Q$ fever

\begin{tabular}{lc}
\hline Variable & Cases (\%) \\
\hline Clinical features & 72 \\
Male sex & 56 \\
Exposure to domestic ruminants & 96 \\
Known valvulopathy & 85 \\
Fever & 54 \\
Heart failure & 48 \\
Hepatomegaly & 36 \\
Splenomegaly & 14 \\
Purpuric rash & 30 \\
Arterial embolism & \\
Laboratory features & 71 \\
Increased erythrocyte sedimentation rate & 49 \\
Anemia & 36 \\
Thrombocytopenia & 32 \\
Increased alanine and aspartate aminotransferases & 27 \\
Increased alkaline phosphatase &
\end{tabular}

Data from references 5 and 37 to 40

all cases of endocarditis in Nova Scotia (3). Cases of $Q$ fever have also been reported in other provinces, including Ontario, Quebec, Newfoundland, New Brunswick and Prince Edward Island (11-16). Seropositivity of more than $15 \%$ of blood donors in Manitoba indicated that unrecognized $Q$ fever may also be present in western Canada (15). To date, no previous case of $Q$ fever has been reported in the province of Alberta.

While acute $Q$ fever is common, only a very small proportion of individuals develop chronic infection, which usually manifests as endocarditis. In a cohort of 1569 patients from France diagnosed with acute $Q$ fever, only 12 individuals developed endocarditis (17). Chronic infection occurs almost exclusively in two groups: those with pre-existing valvular heart disease and those who are immunocompromised $(18,19)$. In one recent series, patients with a valvulopathy and acute Q fever had a 38.7\% chance of going on to develop endocarditis (17). Patients with valvular prostheses are at the greatest risk (19). The median time to development of chronic $Q$ fever was six months following acute infection, although a latent period of up to 20 years has been reported (20). Men account for the majority of cases (75\% in one review [5]), no doubt due to the influence of occupational exposure. Among cases in the immunocompromised population, active malignancy is the biggest risk factor for developing $Q$ fever endocarditis. Whether the malignancy itself or its treatment predisposes to chronic infection is unknown (21). Additionally, numerous cases of endocarditis have been observed in individuals infected with HIV (22).

The clinical presentation of chronic $Q$ fever is insidious and lacks many of the typical features of subacute, bacterial endocarditis. As a result, there is often a significant delay in diagnosis. Despite increasing awareness, recent studies demonstrate a mean delay of seven months from symptom onset to diagnosis (5). The majority of cases present with congestive heart failure due to valvular dysfunction (5). Unlike typical cases of endocarditis, fever is absent in a significant proportion and is frequently intermittent or low grade (17). Although embolic phenomena have been reported in up to one-third of cases, these are usually limited to advanced disease (23). Immune complex glomerulonephritis is frequent and usually
TABLE 2

Serological diagnosis of $Q$ fever

\begin{tabular}{lcccc}
\hline Diagnosis & $\begin{array}{c}\text { Phase I } \\
\text { antibody titre }\end{array}$ & $\begin{array}{c}\text { Phase II } \\
\text { antibody titre }\end{array}$ & $\begin{array}{c}\text { Sensitivity } \\
\mathbf{( \% )}\end{array}$ & $\begin{array}{c}\text { Specificity } \\
\text { (\%) }\end{array}$ \\
\hline Unlikely Q fever & - & $\lg \mathrm{I} \leq 1: 100$ & - & - \\
Acute Q fever & - & $\operatorname{lgG} \geq 1: 200$ & 81.9 & 96.1 \\
& & $\operatorname{lgM} \geq 1: 50$ & 67.2 & 98.8 \\
Chronic Q fever & $\lg \mathrm{Ig} \geq 1: 800$ & - & 100.0 & 99.6 \\
\hline Ig Immunoglobulin & & &
\end{tabular}

Ig Immunoglobulin

manifests as microscopic hematuria (24). A purpuric rash can also be found in a minority of patients (5).

There are many peripheral manifestations of $Q$ fever endocarditis that can provide essential clues as to the diagnosis. Specifically, hepatomegaly and splenomegaly are evident on physical examination in up to one-half of cases (4). Mild hepatitis is usually seen concurrently on biochemical analysis, with elevation of both aminotransferases and alkaline phosphatase. Furthermore, a systemic inflammatory syndrome is seen in most patients, manifested in part by a significantly elevated ESR (18). Many hematological abnormalities are also observed in up to $50 \%$ of cases, with the most frequent being anemia (5). Less commonly observed abnormalities include leukocytosis, leukopenia and thrombocytopenia. A recent report noted that many of the clinical and biochemical abnormalities noted above are absent earlier in the course of chronic Q fever (5). Specifically, the incidence of hematological abnormalities and elevated ESRs were significantly reduced in cases diagnosed within a mean of six months, compared with cases diagnosed at a mean of 18 months. The clinical and laboratory findings of chronic $Q$ fever are summarized in Table 1.

Echocardiography, usually the mainstay of diagnostic imaging in endocarditis, is of limited value in chronic $Q$ fever. In fact, transthoracic echocardiography reveals abnormalities in only $12 \%$ of cases (4). This is due, in large part, to the small size and nodular shape of the typical vegetations (25). While transesophageal echocardiography is far superior in demonstrating lesions, it too has significant limitations. In a recent series, only five of 15 cases had vegetations visible on transesophageal echocardiography, and a further case had a visible abscess (5).

The diagnosis of $Q$ fever endocarditis is hampered by the inability to culture $\mathrm{C}$ burnetii using routine media. As a strict obligate intracellular bacterium, it can only be cultured in living cell lines, most commonly using the shell vial method (26). However, the lack of sensitivity and cumbersome nature of current culture methods has largely limited their use to research laboratories. The diagnosis of chronic $\mathrm{Q}$ fever, therefore, relies on serological testing (Table 2). Chronic $Q$ fever is characterized by increased titres against the phase I antigen. An immunoglobulin $G$ anti-phase I antibody titre of 1:800 or greater has a specificity of $99.6 \%$ for the diagnosis of chronic Q fever $(27,28)$. In fact, the Duke criteria (29) have been modified to included this serological cutoff as a major criterion for the diagnosis of endocarditis.

The gold standard for the diagnosis of infective endocarditis remains the pathological demonstration of organisms on the heart valve. Here too, the diagnosis of $Q$ fever endocarditis remains challenging. In a recent series, only $18 \%$ of cases showed the typical histological features of endocarditis, specifically, vegetations, inflammatory infiltrates and significant 
tissue destruction (30). More commonly, only extensive fibrosis and calcification were evident, which were difficult to discern from degenerative changes. $\mathrm{C}$ burnetii itself is not visible within macrophages on routine hematoxylin and eosin staining, and even special staining is unlikely to reveal the organism. Immunohistochemistry and polymerase chain reaction are more successful in demonstrating the presence of the organism; however, polymerase chain reaction has a sensitivity of only $75 \%(30)$.

The clinical course of $Q$ fever endocarditis is often severe. The mortality among cases reported before 1987 was 37\% (5). The poor prognosis is believed to be due, in large part, to significant delays in diagnosis, which postpone appropriate antimicrobial therapy. This is supported by a recent series in which a mortality rate of only $10 \%$ was observed in cases diagnosed a mean of six months after presentation (5).

The optimal treatment of $Q$ fever endocarditis has not been completely defined, in part due to the difficulty of its culture and the inapplicability of conventional antibiotic susceptibility assays. In vitro studies, using chronically infected cell cultures (which better mimic the conditions of chronic $Q$ fever), have shown doxycycline, chloramphenicol, rifampin and quinolones to be active against $C$ burnetii $(31,32)$. Whether these antibiotics are truly bactericidal or simply bacteriostatic depends on the cell culture model used. In the model developed by Raoult et al (32), only doxycycline in combination with chloroquine exhibited bactericidal activity. Data regarding clinical outcomes with respect to various antibiotic regimens consist mainly of case series and nonrandomized studies. Combination therapy using two antibiotics active against C burnetii in vitro appear to be successful. Specifically, the combination of a fluoroquinolone with either doxycyline or rifampin were successful in controlling infection, although

\section{REFERENCES}

1. Palmer SR, Young SE. Q-fever endocarditis in England and Wales, 1975-81. Lancet 1982;2:1448-9.

2. Fournier PE, Casalta JP, Habib G, Messana T, Raoult D. Modification of the diagnostic criteria proposed by the Duke Endocarditis Service to permit improved diagnosis of $Q$ fever endocarditis. Am J Med 1996;100:629-33.

3. Marrie TJ. A comparison of $Q$ fever endocarditis with native valve endocarditis. Ann N Y Acad Sci 1990;590:61-7.

4. Maurin M, Raoult D. Q fever. Clin Microbiol Rev 1999;12:518-53.

5. Houpikian P, Habib G, Mesana T, Raoult D. Changing clinical presentation of $Q$ fever endocarditis. Clin Infect Dis 2002;34:E28-31.

6. Marrie TJ, Durant H, Williams JC, Mintz E, Waag DM. Exposure to parturient cats: A risk factor for acquisition of $Q$ fever in Maritime Canada. J Infect Dis 1988;158:101-8.

7. Buhariwalla F, Cann B, Marrie TJ. A dog-related outbreak of Q fever. Clin Infect Dis 1996;23:753-5.

8. Marrie TJ, Haldane EV, Noble MA, Faulkner RS, Martin RS, Lee SH. Causes of atypical pneumonia: Results of a 1-year prospective study. Can Med Assoc J 1981;125:1118-23.

9. Marrie TJ. Q fever, 1979-1987 - Nova Scotia. Can Dis Wkly Rep 1988;14:69-70.

10. Marrie TJ, Raoult D. Q fever - A review and issues for the next century. Int J Antimicrob Agents 1997;8:145-61.

11. Hatchette TF, Hudson RC, Schlech WF, et al. Goat-associated $Q$ fever: A new disease in Newfoundland. Emerg Infect Dis 2001;7:413-9.

12. Hatchette T, Hudson R, Schlech W, et al. Caprine-associated Q fever in Newfoundland. Can Commun Dis Rep 2000;26:17-9.

13. Levesque B, De Serres G, Higgins R, et al. Seroepidemiologic study of three zoonoses (leptospirosis, $Q$ fever, and tularemia) among trappers in Quebec, Canada. Clin Diagn Lab Immunol 1995;2:496-8.

14. LeBer C. Q fever, 1982-1986 - Ontario. Can Dis Wkly Rep 1988;14:70-1. relapse rates were as high as $50 \%$ at discontinuation despite prolonged therapy of up to three years $(33,34)$. More recently, the combination of doxycycline and chloroquine was compared with doxycycline and ofloxacin in a nonrandomized, nonblinded study (35). The doxycycline and chloroquine group experienced markedly fewer relapses; in fact, no relapses were observed after 18 months of treatment. The results of this report indicate that doxycycline and hydroxychloroquine may be the preferred treatment for chronic $Q$ fever.

Regardless of the treatment regimen, close clinical monitoring is required. First, given the insidious nature of the disease, patients should have phase I serology checked at regular intervals to ensure efficacy of the regimen. A phase I immunoglobulin $G$ titre of less than 1:200 has been proposed as the main criterion for a clinical cure (36). Similarly, due to the high relapse rate after discontinuation of antibiotics, patients should be followed closely with clinical assessment, echocardiography and laboratory investigation, including phase I serology, at regular intervals (4).

\section{CONCLUSION}

$Q$ fever endocarditis lacks many of the usual clinical and echocardiographic features common to typical cases of endocarditis. As such, the diagnosis is often significantly delayed or even missed, resulting in significant morbidity and mortality. The systemic features of chronic $Q$ fever can provide essential clues as to the presence of Coxiella species infection. However, it is imperative that physicians maintain a high index of suspicion, especially among patients with valvular disease and those who are immunocompromised. Serological testing for Coxiella species provides rapid and accurate diagnosis of chronic $Q$ fever, allowing for prompt initiation of appropriate antimicrobial therapy and improved clinical outcomes.

15. Marrie TJ. Seroepidemiology of $Q$ fever in New Brunswick and Manitoba. Can J Microbiol 1988;34:1043-5.

16. Duperval R, Voiriot P, Baptiste-Desruisseaux D, Marcoux JA, Mongeau CJ. [Q fever in the eastern townships 1975-1984.] Union Med Can 1986;115:873-6.

17. Fenollar F, Fournier PE, Carrieri MP, Habib G, Messana T, Raoult D. Risks factors and prevention of $Q$ fever endocarditis. Clin Infect Dis 2001;33:312-6.

18. Brouqui P, Dupont HT, Drancourt M, et al. Chronic $Q$ fever. Ninety-two cases from France, including 27 cases without endocarditis. Arch Intern Med 1993;153:642-8.

19. Tobin MJ, Cahill N, Gearty G, et al. Q fever endocarditis. Am J Med 1982;72:396-400.

20. Wilson HG, Neilson GH, Galea EG, Stafford G, O'Brien MF. $Q$ fever endocarditis in Queensland. Circulation 1976;53:680-4.

21. Raoult D, Brouqui P, Marchou B, Gastaut JA. Acute and chronic $Q$ fever in patients with cancer. Clin Infect Dis 1992;14:127-30.

22. Raoult D, Levy PY, Dupont HT, et al. $Q$ fever and HIV infection. AIDS 1993; 7:81-6.

23. Stein A, Raoult D. Q fever endocarditis. Eur Heart J 1995;16(Suppl B):19-23.

24. Korman TM, Spelman DW, Perry GJ, Dowling JP. Acute glomerulonephritis associated with acute $\mathrm{Q}$ fever: Case report and review of the renal complications of Coxiella burnetii infection. Clin Infect Dis 1998;26:359-64.

25. Jortner R, Demopoulos LA, Bernstein NE, et al. Transesophageal echocardiography in the diagnosis of $Q$-fever endocarditis. Am Heart J 1994;128:827-31.

26. Raoult D, Vestris G, Enea M. Isolation of 16 strains of Coxiella burnetii from patients by using a sensitive centrifugation cell culture system and establishment of the strains in HEL cells. J Clin Microbiol 1990;28:2482-4. 
27. Fournier PE, Marrie TJ, Raoult D. Diagnosis of Q fever. J Clin Microbiol 1998;36:1823-34.

28. Dupont HT, Thirion X, Raoult D. Q fever serology: Cutoff determination for microimmunofluorescence. Clin Diagn Lab Immunol 1994;1:189-96.

29. Li JS, Sexton DJ, Mick N, et al. Proposed modifications to the Duke criteria for the diagnosis of infective endocarditis. Clin Infect Dis 2000;30:633-8.

30. Lepidi H, Houpikian P, Liang Z, Raoult D. Cardiac valves in patients with Q fever endocarditis: Microbiological, molecular, and histologic studies. J Infect Dis 2003;187:1097-106.

31. Yeaman MR, Mitscher LA, Baca OG. In vitro susceptibility of Coxiella burnetii to antibiotics, including several quinolones. Antimicrob Agents Chemother 1987;31:1079-84.

32. Raoult D, Drancourt M, Vestris G. Bactericidal effect of doxycycline associated with lysosomotropic agents on Coxiella burnetii in P388D1 cells. Antimicrob Agents Chemother 1990;34:1512-4.

33. Cacoub P, Wechsler B, Chapelon C, Thibon M, Godeau P. Q-fever endocarditis and treatment with the fluoroquinolones. Arch Intern Med 1991;151:816,818.
34. Levy PY, Drancourt M, Etienne J, et al. Comparison of different antibiotic regimens for therapy of 32 cases of $Q$ fever endocarditis. Antimicrob Agents Chemother 1991;35:533-7.

35. Raoult D, Houpikian P, Tissot Dupont H, Riss JM, Arditi-Djiane J, Brouqui P. Treatment of $\mathrm{Q}$ fever endocarditis: Comparison of 2 regimens containing doxycycline and ofloxacin or hydroxychloroquine. Arch Intern Med 1999;159:167-73.

36. Raoult D. Treatment of Q fever. Antimicrob Agents Chemother 1993;37:1733-6.

37. Duroux-Vouilloz C, Praz G, Francioli P, Peter O. [Q fever with endocarditis: Clinical presentation and serologic follow-up of 21 patients.] Schweiz Med Wochenschr 1998;128:521-7.

38. Boyle B, Hone R. Q fever endocarditis revisited. Ir J Med Sci 1999;168:53-4

39. Siegman-Igra Y, Kaufman O, Keysary A, Rzotkiewicz S, Shalit I. $Q$ fever endocarditis in Israel and a worldwide review. Scand J Infect Dis 1997;29:41-9.

40. Sanchez-Recalde A, Mate I, Lopez E, et al. [Coxiella burnetii endocarditis: Long-term clinical course in 20 patients.] Rev Esp Cardiol 2000;53:940-6. 